Article available at nttp://Www.parasite-journal.org or nttp://dx.dol.org/10.1051/parasite/1997043217

\title{
LE CONTRÔLE DE LA SCHISTOSOMOSE INTESTINALE De L'île de la MARTINIQUe
}

\author{
SCHLEGEL L*., POINTIER J.P.**, PETITJEAN-ROGET V.***, NADEAU Y.***, BLATEAU A.**** \& MANSUY J.M.*
}

Summary : THE CONTROL OF INTESTINAL SCHISTOSOMIASIS FROM MARTINIQUE ISLAND

The presence of schistosomiasis mansoni is known in Martinique since the beginning of the XXth century. A general survey of the distribution of the disease was carried out in 1977 and showed a mean prevalence of $12 \%$ (coprology and serology taken together) in the whole of the island. Following this survey, an integrated control programme associating sanitary education, detection and treatment of patients and improved sanitation, was developed. In addition, a biological control programme against the intermediate snail host, Biomphalaria glabrata using the competitor snail,

Melanoides tuberculata, was developed in the transmission sites. The decline of snail populations and of its parasite, as well as a strong reduction of the prevalence in humans were recorded between 1977 and 1996. At the present time, only few cases corresponding to older infections are detected. This epidemiological situation is quite different from that in Guadeloupe island where, in spite of an excellent control programme which was achieved on the Basse-Terre district, an important focus is still functionning on Grande-Terre district with the black rat as host reservoir. Such foci do not exist on Martinique island.

KEY WORDS : schistosomiasis, Schistosoma mansoni, control, Martinique island, Biomphalaria glabrata, Melanoides tuberculata.

\section{Résumé :}

En Martinique, l'existence de la schistosomose intestinale à Schistosoma mansoni est connue depuis le début du XXe siècle. Une étude générale de la répartition de la maladie réalisée en 1977 a montré une prévalence moyenne de l'infection de $12 \%$ pour l'ensemble de l'île (coprologies et sérologies confondues). A la suite de cette enquête, un programme de lutte associant des actions d'éducation sanitaire, le dépistage et le traitement des porteurs de la parasitose et l'amélioration de l'assainissement, a été mis en place. Par ailleurs, un programme de contrôle biologique contre le mollusque hôte intermédiaire Biomphalaria glabrata utilisant un compétiteur, Melanoides tuberculata, a été développé dans les sites actifs de transmission. En parallèle à la réduction des populations de l'hôte intermédiaire et de son parasite, une importante diminution de la prévalence globale de la parasitose chez l'homme a été observée entre 1977 et 1996 Désormais, seuls quelques cas sont encore diagnostiqués, mais il s'agit uniquement d'adultes contaminés longtemps auparavant. Cette situation épidémiologique est tout à fait différente de celle qui est observée dans l'île sœur de la Guadeloupe où bien qu'un excellent contrôle des bassins versants de la Basse-Terre ait été réalisé, un important foyer situé en arrière mangrove de la Grande-Terre fonctionne toujours avec un hôte réservoir, le rat noir. En Martinique, plusieurs enquêtes réalisées dans des milieux similaires ont démontré que de tels foyers n'existent pas.

MOTS CLÉS : schistosomose, Schistosoma mansoni, contrôle, Martinique Biomphalaria glabrata, Melanoides tuberculata.

tion dans les zones agricoles et rurales mais également en milieu semi-urbain. Les schistosomoses urinaires et intestinales affectent au moins 200 millions de personnes (WHO, 1985). Il s'agit de maladies liées au péril fécal dont la fréquence est élevée dans les zones d'habitat insalubre sans assainissement satisfaisant. En Amérique du Sud et aux Antilles, il n'existe qu'une seule affection, la schistosomose intestinale, dont l'agent pathogène est Schistosoma mansoni. En Martinique, la schistosomose a été mise en évidence dès le début du siècle mais une étude générale de la répartition de la maladie n'a été réalisée qu'en 1977 par l'Institut National de la Santé et de la Recherche Médicale (INSERM, 1977). Cette enquête a montré que la prévalence moyenne de l'infection était de $12 \%$ pour l'ensemble de l'île (sérologie et examens de selles confondus) avec de fortes disparités selon les zones (au Nord de l'île, la prévalence pouvait atteindre $40 \%$ ). A la suite de ce travail, un inventaire général des sites

\footnotetext{
* Laboratoire Départemental d'Hygiène, BP 628, 97262 Fort-deFrance Cedex.

${ }^{* *}$ Laboratoire de Biologie Marine et Malacologie, École Pratique des Hautes Études, Centre de Biologie et d'Écologie Tropicale et Méditerranéenne, UMR 5555 du CNRS, 52, avenue de Villeneuve, 66860 Perpignan Cedex.

*** Service de Lutte contre les Parasitoses Intestinales, DDASS de Martinique, Boulevard Pasteur, 97200 Fort-de-France Cedex.

${ }^{* * * *}$ Santé et Environnement, DDASS de Martinique, Boulevard Pasteur, 97200 Fort-de-France Cedex.

Correspondance : J.P. Pointier.

Tél. : 0468662055 - Fax : 0468503686.
} 
de transmission fut entrepris et un plan de lutte contre les parasitoses intestinales développé par la Direction Départementale des Actions Sanitaires et Sociales (DDASS) de Martinique. Dans les départements d'outremer, ces programmes sont réglementés par le décret 73-705 du 10 juillet 1973 qui prévoit des actions dans les domaines de l'éducation sanitaire, du dépistage des parasitoses et de l'amélioration des adductions d'eau. En Martinique, des actions pour la santé ont été développées bien avant 1973 mais ce n'est qu'en 1978 qu'une action coordonnée fut mise en place avec la création d'un comité départemental de lutte contre les parasitoses intestinales et d'un service de lutte intégré à la DDASS. Le programme comprenait le dépistage et la prise en charge médicale des porteurs de parasites (avec déclaration des cas survenant chez les enfants de moins de 10 ans), la diffusion d'informations sanitaires et médicales à l'attention des populations exposées et la surveillance des collections d'eau de l'île avec un recensement des gîtes à planorbes, mollusques hôtes intermédiaires de la bilharziose. Ces actions étaient réalisées en parallèle au développement de l'assainissement individuel et collectif.

Cet article se propose de faire le bilan actuel des actions de lutte qui ont été menées en Martinique contre la schistosomose intestinale à partir des différents rapports et articles scientifiques qui ont été publiés ainsi que des résultats du dépistage des parasitoses intestinales effectuées au cours des dernières années par le Laboratoire Départemental d'Hygiène (LDH).

\section{LES MOLLUSQUES HÔTES INTERMÉDIAIRES}

$\mathrm{E}$ n Martinique deux espèces de planorbes appartenant au genre Biomphalaria sont susceptibles de jouer un rôle dans la transmission de Schistosoma mansoni : Biomphalaria glabrata et $B$. straminea.

Biomphalaria glabrata est signalé dans tous les inventaires malacologiques réalisés entre 1874 et 1967 (Mazé, 1874; Bordaz, 1899; Dreyfuss, 1953; Grétillat, 1967). Selon ces auteurs, il s'agirait d'une espèce commune dans de nombreux types d'habitats de Martinique. Cependant, des inventaires plus complets effectués entre 1972 et 1978 ont montré que cette espèce était relativement rare (Guyard et Pointier, 1979). Ces derniers auteurs n'ont toutefois pas fait figurer dans cet inventaire une catégorie d'habitats bien particulière mais importante du point de vue épidémiologique, les cressonnières. En 1980, ces cressonnières ont fait l'objet d'une enquête spécifique qui a montré la présence de B. glabrata et de B. straminea dans la majorité des sites prospectés (fig. 1)(Guyard et al, 1982; Pointier et Guyard, 1992).
Biomphalaria straminea est signalé pour la première fois en Martinique en 1966 par Grétillat sous le nom de $B$. havanensis. Son introduction dans l'île est peutêtre antérieure à cette date mais dès la fin des années 1970 cette espèce est devenue très commune dans toutes les collections d'eau martiniquaises (Guyard et Pointier, 1979). En 1967, lors d'une prospection dans la rivière du Carbet, Paraense (communication personnelle) récolte $B$. straminea infesté naturellement. Cependant, toutes les enquêtes réalisées ultérieurement n'ont pu mettre en évidence un seul mollusque parasité (Guyard et Pointier, 1979). Cette espèce est bien connue au Brésil où malgré sa faible susceptibilité à $S$. mansoni, elle est responsable de la présence d'importants foyers de schistosomose dans la région du Nord-Est (Lucena, 1963; Barbosa et Figueiredo, 1970). Il est donc difficile d'affirmer qu'en Martinique, B. straminea n'a pas joué de rôle dans la transmission du parasite. Il n'en est pas moins vrai que ce rôle a du être très limité en comparaison de celui joué par B. glabrata.

Toutes les enquêtes malacologiques et cercariométriques effectuées entre 1972 et 1983 montrent que la transmission de la schistosomose est presque totale-

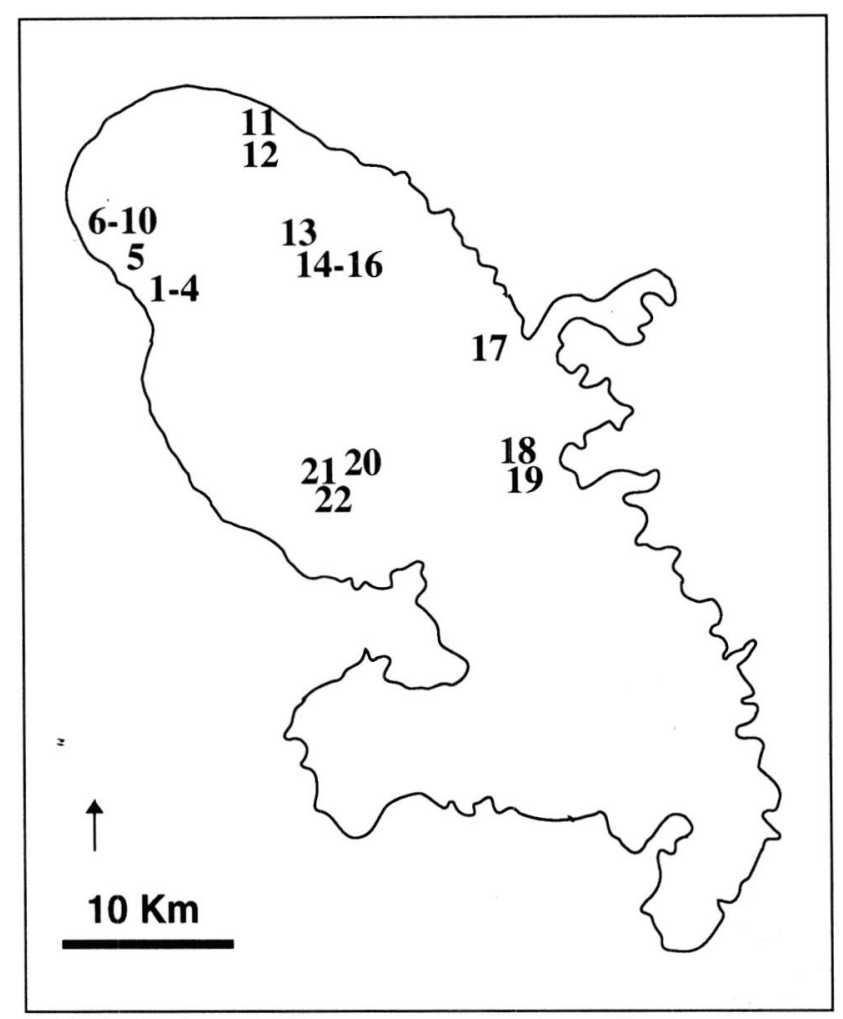

Fig. 1. - Cressonnières de Martinique hébergeant Biomphalaria glabrata et B. straminea en 1980. Le mollusque compétiteur Melanoides tuberculata a été introduit dans ces sites entre 1983 et 1986. 1-4 = Roxelane; 5 = Rivière des Pères; 6-10 = Pointe-la-Mare; $11=$ Habitation Pécoul; 12 = Rivière Falaise; 13 = Bois de l'Union; $14-16=$ Romanet; 17 = Ressource Bassignac; 18 = Haut Roches Carrées; $19=$ Roches Carrées; 20 = Hôtel des Plaisirs; 21-22 = Rivière l'Or. 
ment interrompue en Martinique dès cette époque. Cette interruption, vraisemblablement progressive, remonte à la fin des années 1960 comme le suggère l'infestation des tranches d'âge de la population, tant au niveau général de l'île, que de celui de sites particuliers comme celui du foyer de Pointe la Mare (fig. 2). Trois hypothèses peuvent être avancées pour expliquer cette interruption progressive de la transmission :

1) La raréfaction de l'hôte intermédiaire principal B. glabrata à la suite de l'invasion des collections d'eau par $B$. straminea, espèce mieux adaptée au régime irrégulier des ravines et des rivières et beaucoup moins susceptible au parasite.

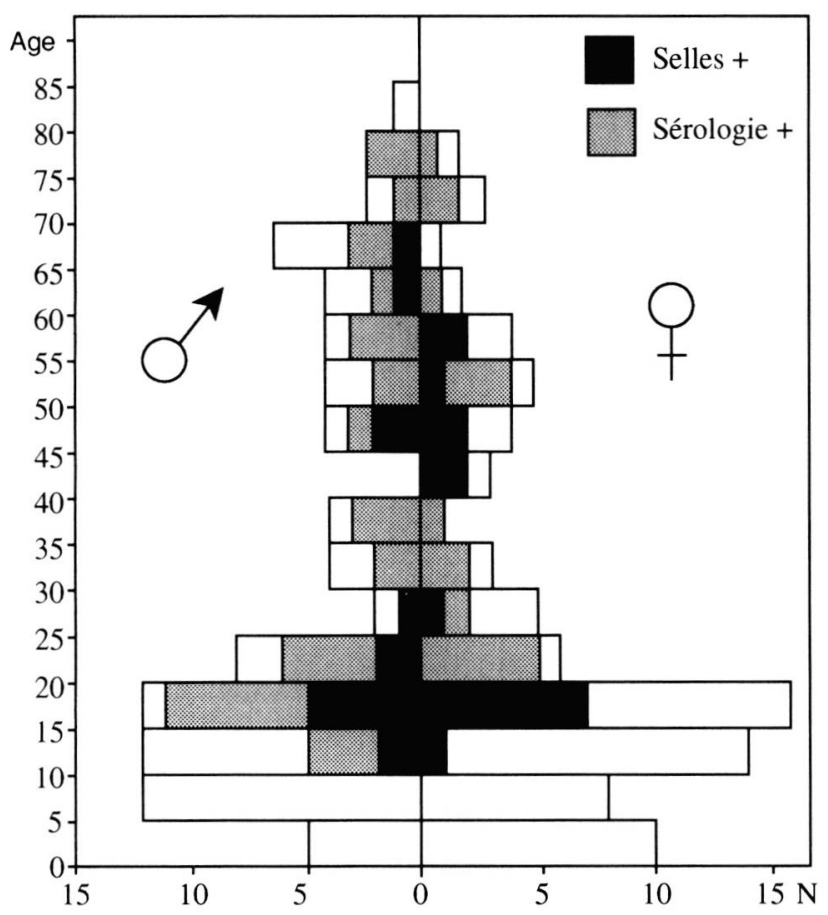

Fig. 2. - Répartition de la schistosomose par tranches d'âge au sein de la population du village de Pointe-la-Mare en 1980. On remarquera l'absence totale d'infestation chez les tranches d'âge de 0 à 10 ans aussi bien en ce qui concerne les sérologies que les analyses de selles. Ce phénomène montre que l'interruption de la transmission remonte à la fin des années 1960 .

2) Une modification du comportement humain vis-àvis de l'eau. La diminution de la fréquence des contacts homme-eau constitue peut-être un facteur qui a joué en faveur d'une interruption de la transmission dans certaines régions. Bien que ce phénomène ait pu être vérifié dans certains cas, il est toutefois difficile d'en apprécier l'importance pour l'ensemble de l'île.

3) Une diminution de la contamination fécale des eaux : le développement des adductions d'eau et des installations sanitaires ont contribué considérablement à la disparition de la contamination fécale des eaux. Celle-ci n'était cependant pas totalement interrompue dans de nombreuses régions comme le montre l'exemple du foyer du quartier du Jardin de Plantes à Saint-Pierre découvert en 1981 (Pointier et al., 1984). Au début des années 1980, la situation était donc tout à fait favorable à la poursuite d'un plan de lutte permettant d'espérer une éradication de la parasitose.

\section{LA LUTTE BIOLOGIQUE}

$\mathrm{P}$ armi les différentes mesures prises pour réduire l'incidence de cette maladie parasitaire, un programme de lutte biologique contre les mollusques hôtes intermédiaires a été mis en place en 1983.

En 1979, le mollusque Thiaridae Melanoides tuberculata était découvert dans la rivière Madame à Fort-deFrance, dans des bassins de l'Habitation Leyritz, et dans une source près de la rivière Falaise (Pointier et McCullough, 1989; Pointier et al., 1993a). Originaire du Moyen Orient et de l'Afrique de l'est, ce mollusque est aujourd'hui répandu dans toute la zone intertropicale où il s'est révélé un excellent compétiteur des planorbes (Prentice, 1983). Il a commencé à envahir la région caraïbe dans les années 1960 et son introduction à la Martinique est liée au commerce des plantes aquatiques qui sont utilisées par les aquariophiles. $M$. tuberculata est essentiellement parthénogénétique et vivipare. Très ubiquiste, il est capable de coloniser de nombreux types d'habitats. L'étude de ses traits de vie montre qu'il a une croissance assez lente, un taux de reproduction faible mais une espérance de vie très longue (Pointier et al., 1991; 1993b). Ces caractéristiques lui confèrent un avantage compétitif dans certains types de milieux vis-à-vis des Biomphalaria. Ceci peut être illustré par les résultats qui ont été obtenus dans les cressonnières de Martinique et qui sont résumés ci-après.

Dès 1983, M. tuberculata est introduit dans le groupe de cressonnières du foyer du Jardin des Plantes de Saint-Pierre qui était alors un site actif de transmission (Pointier et al., 1984). Ce foyer présente les particularités suivantes : un site de transmission très localisé constitué par des cressonnières; une petite population humaine présentant un taux d'infestation de $13 \%$ (coprologies) à $41 \%$ (sérologies); une contamination fécale des eaux faible, ponctuelle et régulière représentée par le déversoir d'un bassin d'épuration non fonctionnel; une forte population de B. glabrata (40 à 256 individus $/ \mathrm{m}^{2}$ ); une faible prévalence d'infestation des mollusques (0,1 à 0,6\%); de faibles densités cercariennes dans les eaux sortant des cressonnières $(0,1$ à 1,2 cercaire par litre); enfin, une très faible contamination de la rivière Roxelane dans laquelle se jettent les eaux ayant traversé les cressonnières. 

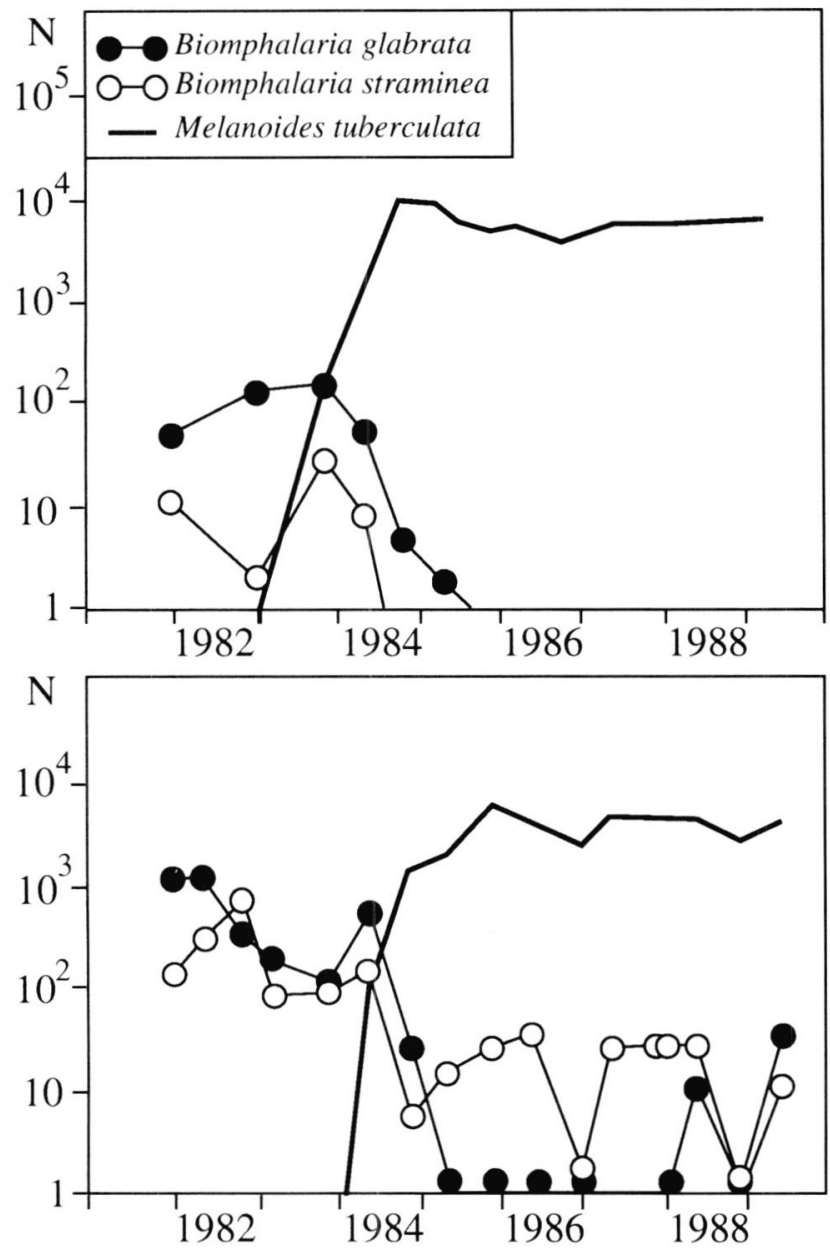

Fig. 3. - Évolution de la densité des populations de Biomphalaria glabrata et B. straminea dans deux cressonnières de Martinique à la suite de l'introduction du mollusque compétiteur Melanoides tuberculata . Cercles noirs = Biomphalaria glabrata ; Cercles clairs $=$ Biomphalaria straminea , Lignes continues $=$ Melanoides tuber culata. On notera la disparition totale des Biomphalaria dans la cressonnière de la Roxelane (en haut) mais leur maintien en faibles densités dans celle de Pointe-la-Mare (en bas). Au total, 22 cressonnières ont fait l'objet d'introductions du compétiteur. Biomphalaria glabrata et B. straminea ont totalement disparu de huit sites; ils sont encore présents en très faibles densités dans sept autres sites; les sept dernières cressonnières correspondent à des cultures qui sont aujourd'hui abandonnées.

En 1985, soit deux années après l'introduction du compétiteur, on observe une disparition quasi totale des deux espèces de planorbes présentes dans le site alors que les densités du mollusque compétiteur se stabilisent à un niveau très élevé (fig. 3). A la suite de ce premier succès, des introductions de $M$. tuberculata ont été réalisées dans les autres groupes de cressonnières qui avaient été répertoriées au début des années 1980. Des évaluations effectuées trois ans après les introductions, puis en 1990 et enfin en 1996, démontrent l'efficacité de la lutte biologique dans ce type d'habitat. En 1996 les deux espèces de planorbes ont totalement disparu de sept sites sur les 22 étudiés. B. glabrata n'est présent que dans un seul site et en très faibles densités (Pointe-la-Mare) tandis que $B$. straminea est toujours présent dans six sites mais également en faibles densités. Les sept autres sites sont des cressonnières dont les cultures ont été abandonnées (Pointier et al., 1989; Pointier et Guyard, 1992).

A partir de 1986, M. tuberculata a commencé à coloniser naturellement de nombreuses collections d'eau et, actuellement, l'espèce a envahi l'ensemble du système hydrographique de la Martinique. Au cours de la même période, les populations de Biomphalaria se sont considérablement raréfiées (fig. 4). En 1996, B. glabrata n'a été récolté que dans deux sites (cressonnières de Pointe-la-Mare et mare de Maupéou) tandis que $B$. straminea est présent de manière sporadique sur l'ensemble de l'île.

\section{PROGRAMME D'ÉDUCATION POUR LA SANTÉ}

$\mathrm{P}$ arallèlement à la lutte biologique contre $B$. glabrata, un programme d'interventions sanitaires et médicales débute à partir de 1978 avec l'objectif de réduire la transmission de l'ensemble des parasitoses intestinales et en particulier celle de la schistosomose. L'équipe pluridisciplinaire chargée de ce programme d'éducation associe un médecin, une infirmière et un sociologue. L'enquête INSERM de 1977 a permis de recenser les zones géographiques les plus atteintes par la schistosomose et de fixer les objectifs prioritaires du programme. Le travail mené par l'équipe de la DDASS peut se décomposer en trois étapes successives: un travail d'approche et d'enquêtes sur les connaissances et les pratiques de la population, suivi des interventions de sensibilisation, d'information et de formation sanitaire et, enfin, l'évaluation de la réussite du programme par la diminution du nombre de parasitoses diagnostiquées.

Les premières enquêtes ont montré que le cycle de la schistosomose était inconnu de la population. Seule la pollution visible (ordures ménagères, déchets encombrants, cadavres d'animaux par exemple) était rendue responsable de la contamination du milieu et la responsabilité de l'homme par le rejet de selles contaminées était ignorée. Toutefois, la schistosomose était la seule parasitose intestinale perçue comme une véritable maladie par opposition aux autres parasitoses qui étaient généralement considérées comme bénignes et non pathogènes. Cette croyance a sans doute amélioré l'efficacité des actions d'éducation.

Les actions du programme d'éducation sanitaire s'adressaient en particulier aux populations les plus exposées (ouvriers agricoles, habitants des quartiers en zones de 

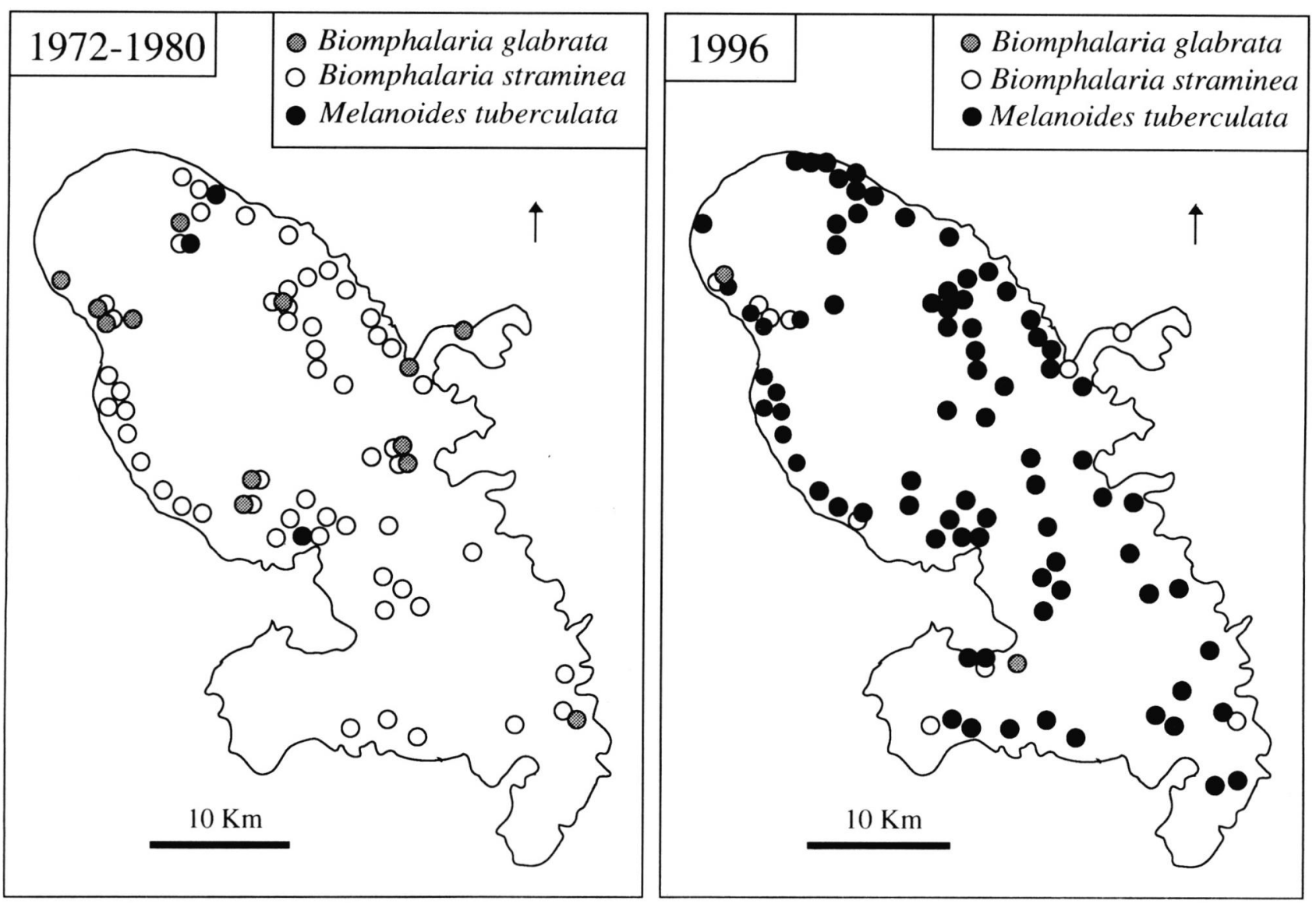

Fig. 4. - Distribution des mollusques hôtes intermédiaires de Schistosoma mansoni en Martinique.

Carte de gauche : synthèse des données recueillies de 1972 à 1980. Entre ces deux dates l'espèce dominante est Biomphalaria straminea (cercles clairs). B. glabrata (cercles gris) est présent dans des cressonnières et quelques autres habitats. En 1979 le mollusque compétiteur Melanoides tuberculata est découvert dans trois sites (cercles noirs). Entre 1983 et 1986, dans le cadre d'un programme de lutte biologique, il est introduit dans 22 cressonnières qui hébergeaient des Biomphalaria.

Carte de droite : résultats de l'inventaire malacologique effectué en 1996; 118 sites hébergent Melanoides tuberculata dont les principaux sont figurés sur cette carte (cercles noirs). En 1996 B. glabrata n'a été récolté que dans deux sites seulement (cressonnière de Pointe-laMare, au nord de l'île et mare de Maupéou au sud, voir les cercles gris). B. straminea quant à lui, est présent de manière sporadique (cercles clairs).

forte endémie) et aux populations jugées réceptives à l'information (enfants scolarisés, jeunes gens lors de leur service militaire, jeunes mères). Le personnel du service de lutte animait des réunions de proximité par petits groupes et favorisait la participation active de toutes les personnes présentes. Des supports spécifiques écrits (affiches, plaquettes d'information et de prévention) et audiovisuels (film "la bilharziose" des Pr Pautrizel et Dr Schodet, réalisé par la Caisse nationale d'assurance maladie des travailleurs salariés et la Caisse générale de sécurité sociale de Guadeloupe) ont aidé à la diffusion de l'information auprès de l'ensemble de la population. L'amélioration des connaissances a ainsi porté sur le cycle du parasite, l'importance du mollusque hôte intermédiaire, et sur le rôle de l'homme dans la transmission. Les pratiques de la population ont également été modifiées pour diminuer la fréquentation des collections d'eau : la pêche aux écrevisses, les baignades et les travaux de blanchissage, traditionnellement réalisés dans la rivière la plus proche, étaient fortement déconseillés. Dans le même temps, le programme expliquait l'importance de la collecte et du traitement des eaux usées. Mais ces campagnes d'éducation ont quelquefois eu un effet néfaste sur l'environnement. En effet, la perception négative des rivières, induite par le message de prévention ("ne pas aller à la rivière"), a rapidement conduit à l'augmentation des rejets (gravats, déchets encombrants, remanants) et à l'assèchement de certains cours d'eau de faible débit. Le message diffusé a alors dû être adapté ("pouvoir bientôt retourner à la rivière") pour permettre de préserver l'environnement.

Désormais, la population connaît le rôle de chaque acteur du cycle parasitaire. Elle sait prendre en charge et gérer le problème des rejets dans le milieu. La réduction des déplacements vers les rivières (réduction 
du nombre de contacts homme-eau potentiellement contaminants) et la bonne utilisation des installations sanitaires ont favorisé la réduction de la transmission parasitaire.

\section{PROGRĖS DE L'ASSAINISSEMENT INDIVIDUEL ET COLLECTIF}

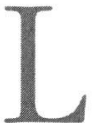

e succès de la lutte contre les parasitoses à transmission oro-fécales est également dépendant de l'amélioration générale de l'hygiène du milieu. Cette amélioration revêt différents aspects : lutte contre l'habitat insalubre, extension des adductions d'eau potable et développement de l'assainissement. L'impact des déclarations en insalubrité sur l'endémie parasitaire n'est pas très important car ces déclarations s'adressent essentiellement aux quartiers urbains alors que les zones rurales sont les plus touchées par les parasitoses.

Si l'installation de réseaux d'eau potable a fortement aidé à la diminution de la transmission de l'ensemble des parasitoses à transmission oro-fécale, dans le cas particulier de la schistosomose intestinale, ces réseaux ont surtout contribué à diminuer la fréquence des contacts avec la rivière.

L'assainissement a pour objet d'assurer l'évacuation des eaux usées ainsi que leur rejet dans les exutoires naturels sans qu'elles puissent souiller les eaux des nappes souterraines, des cours d'eau ou du littoral maritime de manière dangereuse. Il relève de deux techniques différentes (individuel ou collectif) choisies selon des critères techniques ou économiques. En l'absence d'un suivi régulier des installations, la situation actuelle de l'assainissement en Martinique est difficilement comparable aux données antérieures.

L'assainissement individuel est largement répandu en Martinique où il est bien adapté à la dispersion de l'habitat. Depuis 1973, la DDASS, sur le plan technique, et la Caisse d'allocation familiale, sur le plan financier (attribution d'aides incitatives) ont favorisé l'installation de fosses septiques dans les habitations existantes (entre 700 et 800 dossiers sont traités chaque année). Depuis 1993, le nombre d'aides allouées annuellement diminue un peu alors que le nombre de permis de construire d'habitations neuves augmente, assurant le maintien d'un parc fonctionnel étendu.

L'assainissement collectif s'adresse essentiellement à la population agglomérée des villes et à la population de certains lotissements ruraux. En 1986, 22 communes seulement disposent sur une partie de leur territoire d'un réseau d'assainissement collectif avec une station d'épuration. En 1996, 26 communes du département se sont dotées d'un réseau de collecte des eaux usées et de stations d'épuration et les réseaux existants sont plus étendus qu'auparavant. La capacité épuratoire installée a augmenté de 33\%, passant de 150000 à 195000 équivalent-habitant (Blateau, 1986; Direction de l'Agriculture et des Forêts, 1995, données non publiées). Par rapport à la population totale du département, le taux d'équipement est passé de 44,7 à $54,7 \%$ ce qui semble correct. Toutefois, connaître le taux de raccordement aux différents réseaux est difficile en l'absence d'enquête exhaustive. En 1995, un premier dénombrement avait évalué ce taux à 22,4\% de la population totale (Direction de l'Agriculture et des Forêts, données non publiées). La capacité totale installée donne donc une représentation optimiste de la situation. Toutefois, le réseau d'assainissement collectif semble aujourd'hui adapté aux besoins de la population (Blateau, 1995, données non publiées).

\section{DÉPISTAGE PARASITOLOGIQUE ET ÉVALUATION DE L'EFFICACITÉ DES PROGRAMMES DE LUTTE}

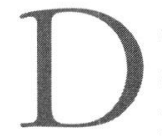
epuis 1977 , le LDH assure en collaboration avec la DDASS, la surveillance des infections parasitaires dans la population martiniquaise (salariés soumis à la visite annuelle de la médecine du travail, femmes enceintes et jeunes enfants suivis dans les centres de protection maternelle et infantile, appelés du contingent lors de leur visite d'incorporation). Il participe également aux études ponctuelles sur la schistosomose intestinale et à l'évaluation de l'efficacité des interventions du comité de lutte contre les parasitoses intestinales (Guyard et al., 1983; Pointier et al., 1984; Mosser, 1986; Mosser et al., 1986).

L'examen parasitologique des selles comprend un examen microscopique à l'état frais et après une étape d'éclaircissement (méthode de Kato). La technique de MIF-concentration permet une recherche plus sensible des faibles parasitismes. Lors de la mise en évidence d'œufs de S. mansoni, le test d'éclosion des miracidia est pratiqué systématiquement pour vérifier leur vitalité. La figure 5 rapporte l'évolution de la prévalence de l'infection à S. mansoni dans la population humaine suivie par le LDH depuis 1966 jusqu'en 1996. Sur la période 1966-1995, la diminution de la prévalence est statistiquement significative ( $x^{2}$ de tendance, $\left.p<0,001\right)$. Les caractéristiques démographiques des patients infectés ne sont malheureusement pas disponibles sur la totalité de la période de surveillance, mais pour les dernières années (1994-1996), la moyenne d'âge des personnes infectées (24 cas) s'établit à 30,8 ans (écarttype: 9,4 ans). Depuis décembre 1984, aucun cas d'infection d'un enfant de moins de 10 ans n'a été déclaré à la DDASS. Dans tous les cas diagnostiqués, les œufs retrouvés étaient viables. 


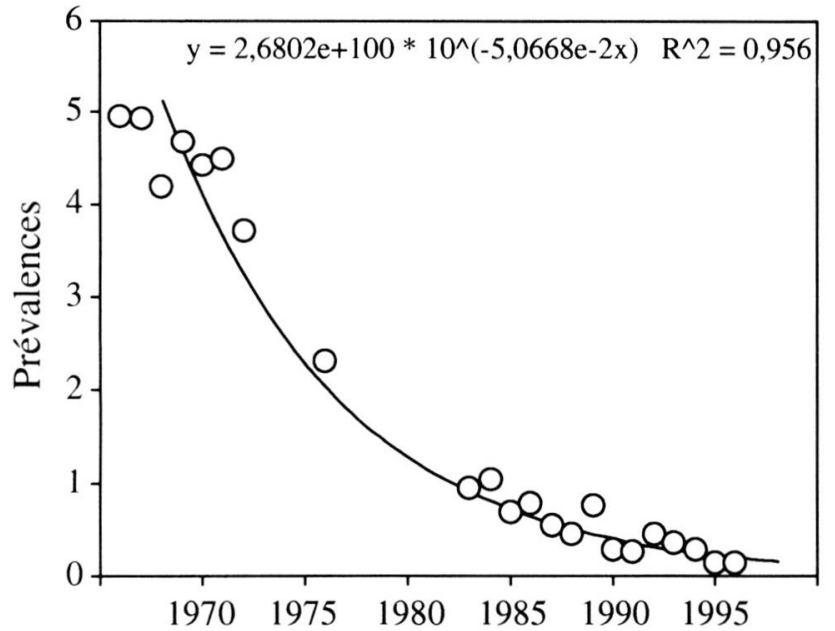

Fig. 5. - Prévalences d'infestation par Schistosoma mansoni dans la population humaine de Martinique suivie par le Laboratoire Départemental d'Hygiène (LDH) entre 1966 et 1996 (examens coprologiques)

Il faut remarquer que les examens parasitologiques des selles pratiqués au LDH sont prescrits souvent chez les patients sans symptomatologie intestinale notable. Toutefois, les résultats obtenus semblent représentatifs de l'ensemble des résultats obtenus à l'échelle du département. En effet, lors de deux confrontations ponctuelles, les quatre principaux laboratoires publics hospitaliers de Martinique avaient évalué la prévalence à S. mansoni à 0,60\% (1988, 17904 examens coprologiques) et 0,27\% (1994-1995 examens coprologiques cumulés) contre respectivement 0,45 et $0,22 \%$ au LDH (Perrin-Bédere et al., 1988; Gardien, 1996, données non publiées).

Les sérologies bilharziennes systématiques (technique d'hémagglutination indirecte), débutées en 1977, ont été abandonnées en 1988 car le taux d'immunité spécifique dans la population, malgré une baisse de 16,8\% à $6,1 \%$, ne variait pas significativement sur la période considérée (Mosser, 1986). En effet, l'immunité détectée correspondant à des infestations anciennes (infestations chroniques ou immunité résiduelle chez des patients traités) ne permet pas un dépistage des porteurs actuels du parasite (Anonyme, 1993).

L'ensemble de ces résultats parasitologiques atteste de l'interruption de la transmission de la bilharziose intestinale en Martinique. Le réservoir humain de parasite est désormais négligeable bien que pour de rares cas diagnostiqués la vitalité des œufs de $S$. mansoni puisse être établie. En effet, la longévité des vers localisés dans le plexus mésentérique inférieur peut atteindre 15 à 30 ans (Anonyme, 1993) : les femelles de S. mansoni venant pondre régulièrement dans la sousmuqueuse intestinale, des œufs viables continuent à être éliminés dans les selles longtemps après la contamination.

\section{TRAITEMENT DES PATIENTS}

$\mathrm{L}$ a volonté d'éradiquer le parasite imposait la prescription d'un traitement antiparasitaire à tous les porteurs du parasite identifiés à l'examen parasitologique. La thérapeutique utilisée a changé en fonction des médicaments disponibles.

Entre 1978 et 1981, le niridazole (Ambilhar ${ }^{\circledR}$ ) était employé en cure de sept jours $(25 \mathrm{mg} / \mathrm{kg} / \mathrm{j})$. Ce traitement entraînait des troubles neurologiques graves (troubles du comportement, agressivité, délire hallucinatoire et convulsions) qui nécessitaient l'hospitalisation des patients et la prescription systématique d'anxiolytiques (Valium ${ }^{\circledR}$ ). La guérison parasitologique n'était pas toujours obtenue et les effets secondaires importants dissuadaient les patients de pratiquer une seconde cure. L'efficacité de ce traitement était donc très réduite.

En 1981, la mise sur le marché de l'oxamniquine $\left(\right.$ Vansil $\left.{ }^{\circledR}\right)$ a radicalement changé la prise en charge des patients. Cet antiparasitaire était prescrit en une seule prise $(15 \mathrm{mg} / \mathrm{kg})$ avec une efficacité et une tolérance remarquable (Gentilini et al., 1981). Il est certain que ce médicament a permis une diminution rapide du nombre de porteurs de $S$. mansoni en améliorant la compliance des patients. Jusqu'en 1987, le Service de Lutte contre les Parasitoses intestinales intégrait les fonctions de dépistage de la maladie, de la prescription du médicament et de sa délivrance. Cette organisation simplifiée facilitait une prise en charge gratuite des patients. Après cette date, et à la suite de la réduction importante du nombre de cas dépistés, le traitement et le suivi des patients ont été confiés aux praticiens libéraux. Toutefois, pour permettre un accès gratuit aux soins, les coûts des consultations médicales et du traitement restaient pris en charge par la DDASS. Un système de déclaration des nouveaux cas était maintenu pour permettre de détecter une éventuelle réapparition de la parasitose.

Depuis 1995, l'arrêt de la commercialisation du Vansil ${ }^{\circledR}$ a conduit au remplacement de ce produit par le praziquantel (Biltricide ${ }^{\circledR}$ ) pour le traitement de la schistosomose intestinale ( $40 \mathrm{mg} / \mathrm{kg}$ répartis en deux prises le même jour).

\section{CONCLUSION}

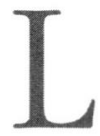
'absence de nouveau cas de schistosomose intestinale dans notre département souligne l'excellente réussite du programme de lutte contre cette parasitose. L'éducation sanitaire a indéniablement contribué à la réduction du nombre de parasitoses liées au péril fécal et en particulier de la schistosomose intestinale. Cette disparition a été accélérée 
par l'amélioration des adductions d'eau et le développement de l'assainissement. La raréfaction du mollusque hôte intermédiaire B. glabrata, conséquence des programmes de lutte biologique menés dans les cressonnières ainsi que de l'invasion naturelle de l'ensemble du réseau hydrographique martiniquais par les compétiteurs, a cependant été l'élément décisif qui a permis le contrôle de $S$. mansoni par la rupture du cycle parasitaire.

La schistosomose intestinale est aujourd'hui bien contrôlée en Martinique mais il est probable que des porteurs d'œufs de $S$. mansoni continueront à être dépistés pendant encore quelques années. Ces rares cas constitueront les stigmates de la situation épidémiologique antérieure lorsque cette parasitose était endémique en Martinique. Malgré l'application de mesures similaires, la situation est tout à fait différente aujourd'hui dans l'île sœur de la Guadeloupe. En effet, si le programme de lutte intégrée mis en place dès la fin des années 1970 a permis un excellent contrôle des bassins versants de la Basse-Terre et l'éradication du foyer sylvestre du Grand Étang, la transmission du parasite est toujours effective dans le foyer d'arrière mangrove de la Grande-Terre (Pointier et al., 1991; Pointier et Théron, 1995; Théron et Pointier, 1995). Ce foyer a la particularité de fonctionner avec un hôte réservoir, le rat noir Rattus rattus dont les populations sont restées très fortement infestées en dépit d'un essai de lutte biologique contre le mollusque hôte intermédiaire et d'un dépistage systématique des habitants de cette région (Théron et al., 1992; Pointier et al., 1993). En Martinique, heureusement, de tels habitats sont extrêmement limités et plusieurs enquêtes réalisées au début des années 1980 sur les populations de rats dans deux sites favorables à un tel type de transmission se sont révélées négatives (Marécages de l'Anse Rivière et du Quartier Boisneuf).

\section{RÉFÉRENCES}

Anonyme. Bilharzioses. In: Médecine tropicale, Gentilini (éd.), Flammarion Médecine-Sciences, Paris, 1993, 221-236

Barbosa F. \& Figueiredo T. Susceptibility of the snail intermediate hosts of schistosomiasis from northeastern Brazil to the infection with Schistosoma mansoni. Revista do Instituto de Medicina Tropical de São Paulo, 1970, 12, 198206.

Blateau A. Le point sur l'assainissement collectif à la Martinique. In: La santé en Martinique, DDASS Martinique, Fortde-France, 1986, 198-206.

Bordaz G. Liste des coquilles recueillies à la Martinique. Bulletin de la Société d'Histoire Naturelle d'Autun, 1899, 12, 5-24.
Dreyfuss R. Les planorbes de la Martinique. Bulletin de la Société Française d'Histoire Naturelle des Antilles, 1953, 2, 41-45.

Gentilini M., Danis M., Petit P., Meunier Y., Brucker G., NeIsSon-Vernant E. \& ARnaud J.P. Traitement de la bilharziose intestinale, évaluation de l'oxamniquine à partir de 172 observations. Médecine et Maladies Infectieuses, 1981, 11, 120.

Grétillat S. Prospection malacologique aux Antilles françaises. Observations sur l'écologie et l'élevage au laboratoire de Lymnaea cubensis. Revue d'Élevage et de Médecine Vétérinaire des Pays Tropicaux, 1967, 2, 279-289.

GUYARD A. \& POINTIER J.P. Faune malacologique dulçaquicole et vecteurs de la schistosomose intestinale en Martinique (Antilles françaises). Annales de Parasitologie, 1979, 54, 193-205.

Guyard A., Pointier J.P., Théron A. \& Dumoutier-Gilles A. La bilharziose en Martinique. CNDP-CRDP Antilles-Guyane, Fort-de-France, 1983, 1-28.

Guyard A., Pointier J.P., Théron A. \& Gilles, A. Mollusques hôtes intermédiaires de la schistosomose intestinale dans les Petites Antilles. Hypothèses sur le rôle de Biomphalaria glabrata et B. straminea en Martinique. Malacologia, 1982, 22, 103-107.

INSERM. Étude de l'endémie parasitaire intestinale en Martinique. INSERM U.165, Le Vézinet, 1979.

LuCENA D.T. Planorbideos transmissores da esquistossomose no nordeste do Brasil. Revista Brasileira e Malariologia Doenças Tropicais, 1963, 15, 13-26.

Maź́ H. Catalogue des coquilles terrestres et fluviatiles recueillies à la Martinique en 1873. Journal de Conchyliologie, 1874, 22, 158-173.

Mosser A. Bilan de l'activité menée chez les militaires 19801986 par le Service de Lutte contre les Parasitoses Intestianles. DDASS Martinique, Fort-de-France, 1986, 1-10.

Mosser A., Neisson-Vernant E. \& Scat Y. État de l'endémie parasitaire intestinale en Martinique en 1986. XX Congrès International des Médecins de Langue Française de l'Hémisphère Américain, Pointe-à-Pitre, 1986.

Perrin-Bédere C., Petitjean-Roget V., Burgos S., Laviolette L. \& Mathurin J. Service de Lutte contre les Parasitoses Intestinales et d'Education pour la Santé. Rapport annuel, DDASS Martinique, Fort-de-France, 1988, 1-41.

POINTIER J.P. \& GUYARD A. Biological control of the snail intermediate hosts of Schistosoma mansoni in Martinique, French West Indies. Tropical Medicine and Parasitology, 1992, 43, 98-101.

Pointier J.P. \& McCullough F. Biological control of the snail hosts of Schistosoma mansoni in the Caribbean area using Thiara spp. Acta Tropica, 1989, 46, 147-155.

Pointier J.P., Guyard A. \& Mosser A. Biological control of Biomphalaria glabrata and B. straminea by the competitor snail Thiara tuberculata in a transmission site of schistosomiasis in Martinique, French West Indies. Annals of Tropical Medicine and Parasitology, 1989, 83, 263-269.

Pointier J.P., Guyard A., Théron A. \& Dumoutier A. Le fonctionnement d'un site de transmission à Schistosoma mansoni en Martinique (Antilles françaises). Annales de Parasitologie, 1984, 59, 589-595. 
Pointier J.P., Thaler L., Delay B. \& Pernot A.F. Invasion of the Martinique island by the parthenogenetic snail Melanoides tuberculata and succession of morphs. Acta Oecologica, International Journal of Ecology, 1993, 14, 33-42.

Pointier J.P., ThÉRON A. Ecology and control of the snail intermediate hosts of trematodes in an heterogenous environment: the Biomphalaria glabrata model in the insular focus of Guadeloupe. Research and Reviews in Parasito$\log$, 1995, 55, 1-13.

Pointier J.P., Théron A. \& Borel G. Ecology of the introduced snail Melanoides tuberculata (Gastropoda: Thiaridae) in relation to Biomphalaria glabrata in the marshy forest zone of Guadeloupe, French West Indies. Journal of Molluscan Studies, 1993, 59, 421-428.

Pointier J.P., Théron A., Imbert-Eestablet D. \& Borel G. Eradication of a sylvatic focus of Schistosoma mansoni using biological control by competiror snails. Biological Control, 1991, 1, 244-247.

Pointier J.P., Toffart J.L. \& Lefevre M. Life tables of freshwater snails of the genus Biomphalaria (B. glabrata, B. alexandrina, B. straminea) and of one of its competitor Melanoides tuberculata under laboratory conditions. Malacologia., 1991, 33, 43-54.

PRENTICE M.A. Displacement of Biomphalaria glabrata by the snail Thiara granifera in field habitats in St Lucia, West Indies. Annals of Tropical Medicine and Parasitology, 1983, 77, 51-59.

Théron A. \& Pointier, J.P. Ecology, dynamics, genetics and divergence of trematode populations in heterogenous environments: the model of Schistosoma mansoni in the insular focus of Guadeloupe. Research and Reviews in Parasitiology, 1995, 55, 49-64.

Théron A., Pointier J.P., Morand S., Imbert-Establet D. \& BOREL G. Long-term dynamics of natural populations of Schistosoma mansoni among Rattus rattus in patchy environment. Parasitology, 1992, 104, 291-298.

WHO. The control of schistosomiasis. WHO. Technical Report Series, 1985, 728.

Reçu le 22 janvier 1997 Accepté le 9 mai 1997 